\title{
The Photometry Pipeline of the Watcher Robotic Telescope
}

\author{
A. Ferrero, ${ }^{1}$ L. Hanlon, ${ }^{1}$ R. Felletti, ${ }^{1}$ J. French, ${ }^{1}$ G. Melady, ${ }^{1}$ S. McBreen, ${ }^{1}$ P. Kubánek, ${ }^{2}$ \\ M. Jelínek, ${ }^{2}$ B. McBreen, ${ }^{1}$ P. Meintjes, ${ }^{3}$ J. Calitz, ${ }^{3}$ and M. Hoffman ${ }^{3}$ \\ ${ }^{1}$ Space Science Group, School of Physics, University College Dublin, Ireland \\ ${ }^{2}$ Instituto de Astrofísica de Andalucía, C/ Bajo de Huétor 50, ES 18008 Granada, Spain \\ ${ }^{3}$ Physics Department, University of the Free State, Bloemfontein 9301, South Africa
}

Correspondence should be addressed to A. Ferrero, alejandro.ferrero@ucd.ie

Received 30 June 2009; Accepted 2 December 2009

Academic Editor: Alberto J. Castro-Tirado

Copyright (C) 2010 A. Ferrero et al. This is an open access article distributed under the Creative Commons Attribution License, which permits unrestricted use, distribution, and reproduction in any medium, provided the original work is properly cited.

\begin{abstract}
The Watcher robotic telescope was developed primarily to perform rapid optical follow-up observations of Gamma-Ray Bursts (GRBs). Secondary scientific goals include blazar monitoring and variable star studies. An automated photometry pipeline to rapidly analyse data from Watcher has been implemented. Details of the procedures to get image zero-point, source instrumental measurement, and limiting magnitude are presented. Sources of uncertainty are assessed and the performance of the pipeline is tested by comparison with a number of catalogue sources.
\end{abstract}

\section{Introduction}

Robotic telescopes offer full automated control of an observatory and its instruments. The key ability of a robotic system is that it can observe without any human intervention. The system generates its own observing schedule according to the priority established from user-specified criteria, such as the altitude of the target. Scheduling is flexible, responding to new requests or events as necessary, or to feedback from environmental monitoring sensors. The system can also be accessed and controlled remotely if required and provide remote users with access to data.

A large proportion of the effort in developing new ground-based astronomy facilities has traditionally been directed towards building larger aperture telescopes. However, at the other end of the scale, an increasing number of scientific programmes are being carried out by smaller instruments operated in an automated or robotic manner. Research goals requiring long time baselines or rapid response times are hampered by the inflexibility and limited availability of observing time at large telescopes. Considerable effort is therefore currently being devoted to developing robotic systems better suited to the specific scientific requirements of such programmes. Robotic telescopes have become increasingly sophisticated due to a variety of technological advances, in particular the Internet, the widespread availability of powerful, affordable computers since the early 1990s and the increasing sophistication of the amateur community, which has opened up new markets for manufacturers of components such as telescope mounts. Reviews of the technological and scientific advances made in robotic telescope astronomy at various times since the mid1990s are given in [1-4].

The main advantage of robotic telescopes lies in their flexibility, which allows variability to be studied in ways that are not possible with traditional facilities [5]. Studies of active galactic nuclei [6], variable stars [7], and extrasolar planetary transits [8] greatly benefit from long-term monitoring programmes that robotic facilities can carry out so effectively. In addition, their rapid, automated response capabilities make them ideally suited to observations of transient sources like supernovae and $\gamma$-ray bursts (GRBs). Whereas manually operated telescopes can respond on timescales of minutes at best, robotic telescopes can begin observing the target in seconds, opening up a previously unexplored regime in the time-domain study of GRBs [9]. Other areas in which robotic telescopes excel are cosmological surveys [10] and calibration tasks such as extinction monitoring. 


\section{The Watcher Robotic Telescope}

The goal of the Watcher project was to develop a fully robotic optical telescope system capable of responding to GRB alerts generated by orbiting satellites, in particular the Swift and INTEGRAL missions. The main design drivers from the science point of view were that the system should be fast and sensitive enough to explore associated optical emission in the early-time regime, tens of seconds or less after the initial trigger, ideally while the burst is still active in $\gamma$-rays. The technical challenges in carrying out such observations have meant that very early-time optical emission had been very sparsely studied in the pre-Swift era, and as a result poorly understood. The unprecedented rate, speed, and accuracy of GRB positions from Swift [11] and INTEGRAL [12] allows for extensive study of their early optical emission. While a number of robotic telescopes dedicated to GRB follow-ups were already in operation, a world-wide network of such instruments is required in order to maximise coverage of the night sky and gather as much early-time optical data as possible. A suitable instrument located at an appropriate site could contribute significantly to these studies.

2.1. Boyden Observatory. The site selected for Watcher was the Boyden Observatory situated at an altitude of $1387 \mathrm{~m}$ in South Africa's Highveld region, $26 \mathrm{~km}$ from the city of Bloemfontein at coordinates $29^{\circ} 02^{\prime} 20^{\prime \prime}$ South, $26^{\circ} 24^{\prime} 20^{\prime \prime}$ East. Boyden was initially established as Harvard University's southern observatory in Peru in 1889 and was relocated to South Africa in 1927. After a number of years of being operated solely by Harvard, the observatory was subsequently run by an international consortium, including Ireland's Dunsink Observatory, until 1976, when it was presented to the University of the Free State (UFS). Following some years of decline, Boyden has been revitalised in recent years thanks to the efforts of the University's Physics Department and the Friends of Boyden organisation. The original $1.5 \mathrm{~m}$ telescope has been refurbished and is actively engaged in research. Construction of the Boyden Science Centre Auditorium was completed not long before Watcher was installed, providing space for talks as well as a night sky viewing platform on its roof. The Observatory has a very active educational and public outreach program, frequently holding open evenings and school visits which make use of the refurbished $13^{\prime \prime}$ refractor, the solar telescope, and Watcher.

Several factors contributed to this choice of site. In order to maximise opportunities for observing GRB optical counterparts from the ground, a globally distributed network of instruments which can provide round the clock coverage of the entire night sky is required. Since sky coverage with dedicated robotic telescopes at southern latitudes is relatively sparse, and only one other system is in operation in Africa (ROTSE-IIIc at the HESS site in Namibia), Boyden was a natural choice. Atmospheric conditions at the site are generally very stable, particularly in the winter as the region's dominant high-pressure systems act as a barrier to moisture streams. However during the summer high surface temperatures and streams of warm moisture-laden air from the Indian Ocean can result in cloud formation and occasional violent thunderstorms [13]. Though the site is at a relatively high altitude and is sufficiently remote to avoid major light pollution problems, it is still very accessible, which facilitates installation, upgrades, and any necessary maintenance. The seeing is typically $\sim 2^{\prime \prime}$, about 2.5 times the pixel scale.

2.2. Instrument Overview. Watcher began observing in March 2006, and operated in fully automated mode from May until early October that year, when some control electronics were damaged by a lightning strike. It was back in operation in January 2007 until October 2008, when a major upgrade began. The optical tube is a $40 \mathrm{~cm}$ ClassicalCassegrain on a Paramount ME robotic mount. The detector is an Apogee AP6e CCD, with a KAF-1001e $1 \mathrm{k} \times 1 \mathrm{k}$ chip. The field of view is $14.5^{\prime} \times 14.5^{\prime}$, with a plate scale of $0.85^{\prime} /$ pixel. Bessell BVRI filters are housed in an Optec IFW filter wheel, and automatic focussing is provided by a Robofocus stepper-motor, located at the secondary mirror. A Davis Vantage Pro weather station monitors weather conditions and can shut down Watcher in the event of rain or thick cloud cover. The motorised roll-back roof mechanism is controlled by custom-made electronics. Watcher is controlled by two Linux PCs running RTS2 software, a powerful, open-source package for complete management of a remote observatory [14]. This software automatically manages all the hardware, schedules observations of targets in a local database and can respond to GCN alerts for rapid GRB follow-up observations. A full description of Watcher is in preparation [15].

\section{The Photometry Pipeline}

A customised pipeline has been implemented for the consistent, reliable, and rapid analysis of Watcher data. Rapid dissemination of new information is particularly important in the case of GRB observations where newly identified afterglows can act as triggers for Target of Opportunity override programmes on larger telescopes.

A flow-chart of the steps involved in the photometry pipeline is shown in Figure 1. Apart from the CCD reduction, for which IRAF is used, all procedures are implemented in Matlab/Octave, providing greater flexibility in the data processing.

3.1. CCD Reduction. The CCD reduction is accomplished in IRAF in the usual way according to

$$
I_{\text {red }}=\frac{I_{\text {raw }}-I_{\text {dark }}}{F},
$$

where $I_{\text {red }}$ is the reduced image, $I_{\text {raw }}$ is the raw image, $I_{\text {dark }}$ is the dark image. $F$ is the flatfield correction factor, calculated according to

$$
F=\operatorname{median}\left(\frac{I_{\mathrm{ff}, i}-I_{\mathrm{dark}}}{\left\langle I_{\mathrm{ff}, i}-I_{\mathrm{dark}}\right\rangle}\right),
$$

where $I_{\mathrm{fff}, i}$ is the $i$ th-flatfield image and the median is obtained over the number of flatfields taken for a given filter. It is 


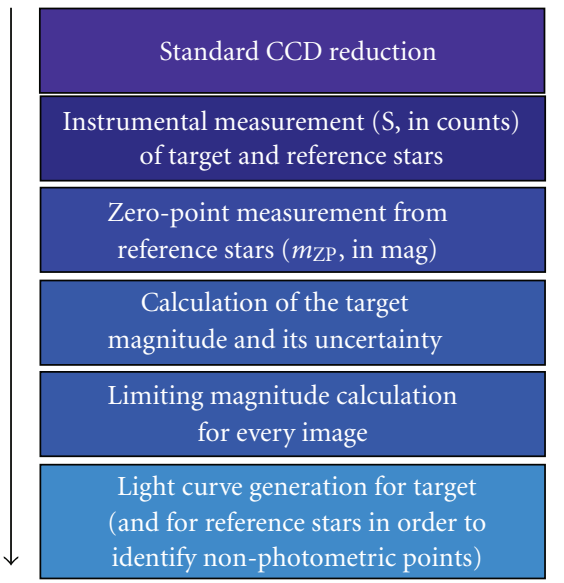

FIgURE 1: Flowchart of Watcher's photometry pipeline.

important that the target and dark images are taken at the same temperature (within $\pm 1^{\circ} \mathrm{C}$ ) to keep the dark current and bias values sufficiently stable. In the event that the flatfield image is of poor quality, as can happen in the case of some GRB observations, adequate photometry can still be obtained by eliminating the flatfield correction since the nonuniformity of the image field is only $\sim 2 \%$.

3.2. Source Magnitude and Uncertainty Calculation. The source magnitude, $m_{\text {target }}$, is calculated according to

$$
m_{\text {target }}=m_{\mathrm{ZP}}-2.5 \log S,
$$

where $m_{\mathrm{ZP}}$ is the zero-point uncertainty, $S$ is the source count and $m_{\text {ins }}=-2.5 \log S$ is the instrumental magnitude of the source.

The uncertainty on the calculated magnitude is then given by

$$
u(m)=\sqrt{u_{\mathrm{ZP}}^{2}+1.1788\left(\frac{u_{\mathrm{S}+\mathrm{sky}}^{2}+u_{\mathrm{sky}}^{2}}{S^{2}}\right)},
$$

where $u_{Z P}$ is the zero-point uncertainty, $u_{S+s k y}$ is the source plus sky uncertainty, and $u_{\text {sky }}$ is the sky uncertainty. Equations (3) and (4) are applied to all sources of interest (targets and reference stars). The measured magnitudes of a number of reference sources versus catalogue magnitudes for a test Watcher image are shown in Figure 2. The slope of this graph shows that the Watcher clear filter corresponds reasonably closely to the USNO-B1, R2 filter.

3.2.1. Instrumental Measurement of a Source. Conventional aperture photometry is used to calculate $S$ from $(\mathrm{S}+$ sky) sky. The typical aperture radius used is 1.75 FWHM. The uncertainty in the instrumental measurement of the source is given by:

$$
u_{\mathrm{S}}=\sqrt{u_{\mathrm{S}+\mathrm{sky}}^{2}+u_{\text {sky }}^{2}} \text {. }
$$

The standard deviation per pixel $\left(\sigma_{\text {sky }}\right)$ is calculated from the sky annulus pixels. When the value of a pixel deviates

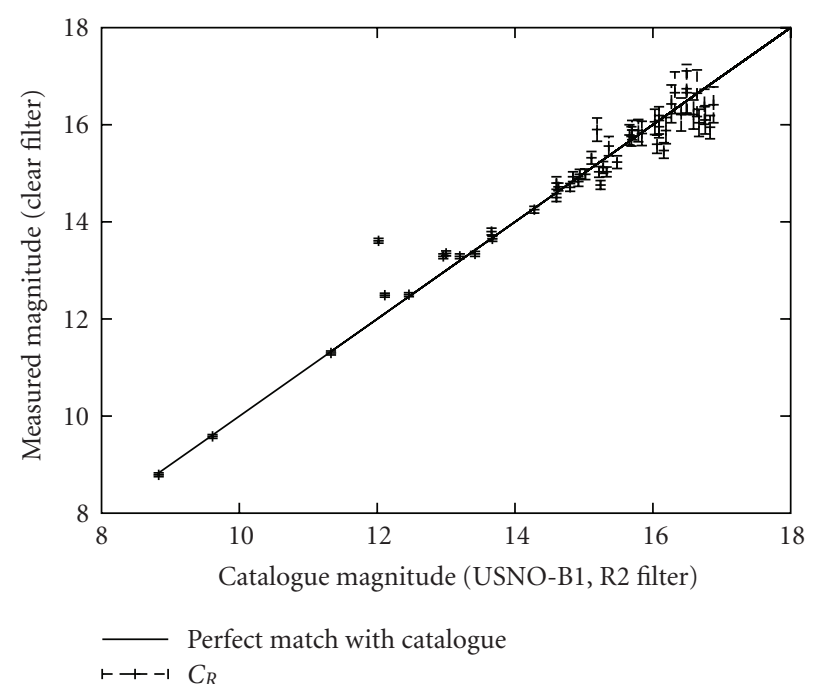

FIGURE 2: Measured magnitudes of reference stars versus catalogue magnitudes for a Watcher test image.

by more than $2 \sigma_{\text {sky }}$ from the sky average, it is rejected in order to avoid contamination of the sky measurement due to neighbouring sources. The uncertainty in the sky measurement is given by

$$
u_{\text {sky }}=\sigma_{\text {sky }} \frac{N_{\mathrm{ap}}}{\sqrt{N_{\mathrm{ann}}}},
$$

where $N_{\text {ap }}$ is the number of pixels in the aperture and $N_{\mathrm{ann}}$ is the number of pixels in the sky annulus. The uncertainty on the source plus sky measurement, $u_{\mathrm{S}+\text { sky }}$, is given by

$$
u_{S+s k y}=\sqrt{\frac{S}{G}+\sigma_{\text {sky }}^{2} N_{\text {ap }}}
$$

where $G$ is the conversion factor of the CCD from counts to electrons. Readout noise is already included in $\sigma_{\text {sky. }}$. If $N_{\text {ann }}$ is large enough, $u_{\text {sky }}$ is negligible compared to $u_{S+s k y}$.

3.2.2. Zero-Point Measurement of an Image. The zero-point is assumed to be constant across the image. This is verified by comparing the temporal evolution of the magnitudes of different stars within an image. Instrumental measurements of all catalogued stars in a radius of 300 arcsec from the target are determined. Instrumental magnitudes are then calculated for each star, where SNR $>10$, from catalogued magnitudes and instrumental measurements. Points deviating by more than $1 \sigma$ are removed from the distribution of calculated zero-points. The standard deviation of the average $\left(\sigma_{\mathrm{ZP}} / \sqrt{N}\right)$ of the new distribution is the uncertainty of the zero-point $\left(u_{\mathrm{ZP}}\right)$. A larger number of stars used for the zero-point determination naturally results in lower uncertainty. No weighting is used in the averaging.

3.3. Limiting Magnitude Measurement. Reliable determination of the limiting magnitude in an image is important, particularly for GRB observations where the source can 


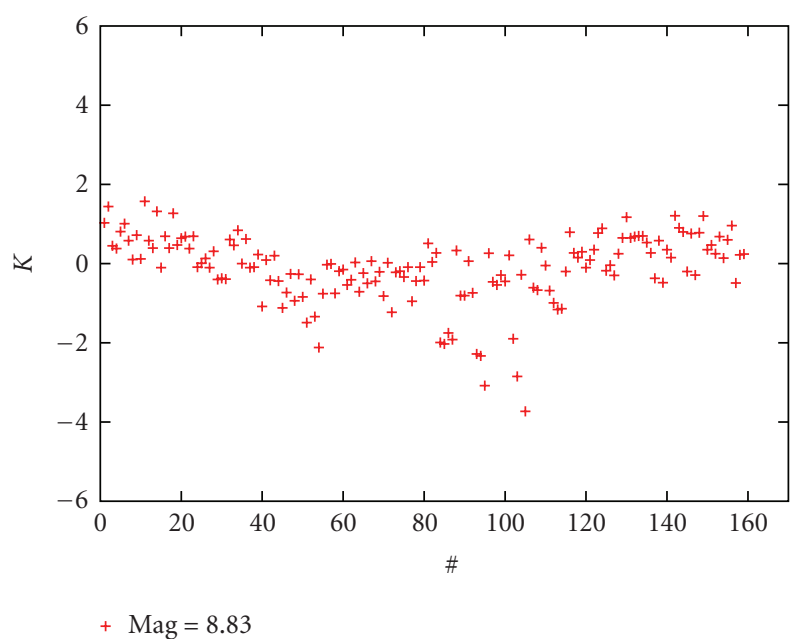

FIGURE 3: Bright source: uncertainty limited by the zero-point measurement.

fluctuate strongly and its brightness may be close to the limit of a small telescope's capabilities.

The limiting magnitude should be expressed as a measurement, since due to the uncertainty in the catalogue values, it is calculated from a distribution.

For all sources with SNR between 1 and 10, $\log$ SNR is linearly fitted to the corresponding catalogue magnitude. From the resultant equation, the limiting magnitude corresponding to a SNR $=2$ is calculated. The uncertainty on the limiting magnitude is then calculated from the uncertainty of the best-fit parameter.

\section{Test of Photometry Performance}

To assess the performance of the pipeline, 159 images of two specific nonvariable stars were selected and the statistical behaviour examined using a $\chi^{2}$ test, with $\chi^{2}=$ $\sum^{N} k^{2}$, where $k=\left(m_{\text {ins }}-m_{\text {average }}\right) / u(m)$. The first star's derived magnitude is 8.83 where the uncertainty is limited by the zero-point measurement. In this case, $\chi^{2} / \nu$ is 0.77 (122.1/158), suggesting that the uncertainty on the zeropoint is slightly overestimated. The second star is a faint source (magnitude $=17.15$ ), whose uncertainty is limited by the instrumental measurement. In this case $\chi^{2} / v$ is 1.03 $(162.4 / 158)$. The index $k$ for both stars is plotted in Figures 3 and 4 as a function of the image number (\#).

\section{Preliminary Results: GRB 080905B}

Watcher observations of GRB 080905B began $44.3 \mathrm{~s}$ after the trigger, with images being acquired without filter (clear, $\left.R_{C}\right)$, with exposures of 10 seconds $(44.3 \mathrm{~s}-656.3 \mathrm{~s}), 30 \mathrm{sec}-$ onds (660.3s-3591.3s), and 60 seconds ( $3718.3 \mathrm{~s}-7264.3 \mathrm{~s})$. The zero-points of the measurements were calculated by comparison with the R2 magnitude in the USNO B.1 catalogue. The 2MASS galaxy, 2MASX J20065732-6233465, is located 4 arcsec from the GRB. The galaxy contribution was subtracted from the data by calculating the flux of

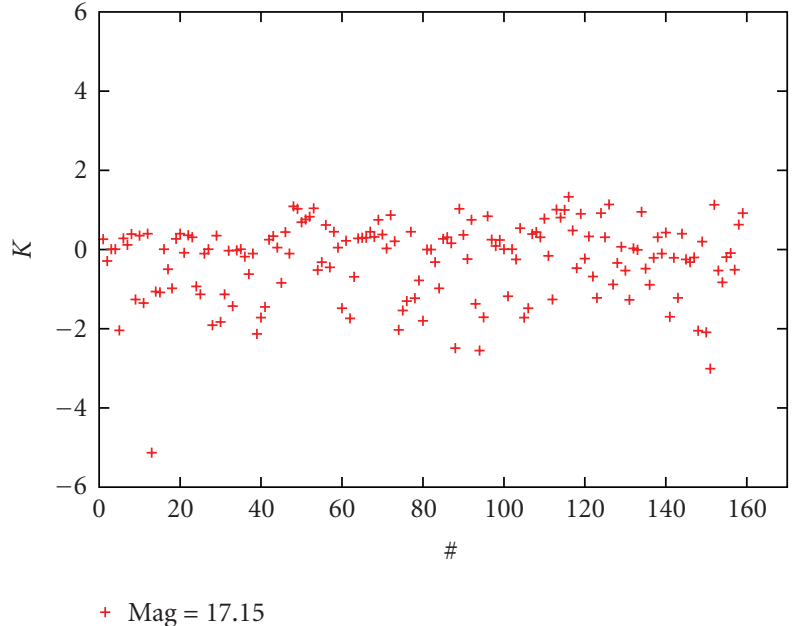

FIGURE 4: Faint source: uncertainty limited by the instrumental measurement.

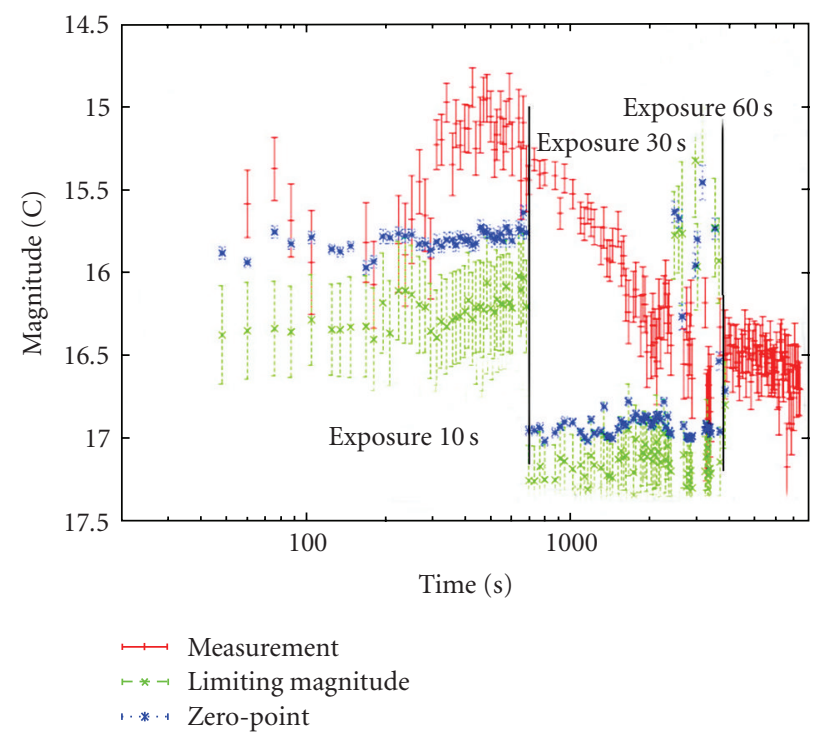

FIGURE 5: The Watcher light curve of the prompt and early afterglow emission from GRB 080905B in the clear filter band with the time quoted relative to the GRB trigger time. The zero-point and limiting magnitude for every exposure are also shown. Since the value of the zero-point is related to the total number of counts per exposure time unit, its temporal evolution is correlated with the limiting magnitude evolution and the exposure time.

photons in the aperture from a set of long exposure images $(120 \mathrm{~s})$ that were acquired a long time after the trigger, when the contribution of the optical transient to the flux was negligible. In the Watcher light curve, the prompt optical emission and optical afterglow are clearly separated (Figure 5). The origin of the peaks in limiting magnitude at around $3000 \mathrm{~s}$ is most likely due to intermittent cloud. The separation of the optical components shows their temporal relationship and relative strength and will be presented in more detail in a future publication [16]. 


\section{Conclusions}

The Watcher photometry software provides a robust, automated pipeline for the automated processing of large sets of CCD data. In particular for GRB observations, it permits limiting magnitudes to be properly calculated for each exposure setting, so that in the absence of an afterglow detection, a reliable upper limit can be rapidly disseminated.

\section{Acknowledgment}

The authors acknowledge the support from the Science Foundation Ireland under Grant 07-RFP-PHYF295.

\section{References}

[1] G. W. Henry and J. A. Eaton, Eds., Robotic Telescopes: Current Capabilities, Present Developments, and Future Prospects for Automated Astronomy, vol. 79 of Astronomical Society of the Pacific Conference Series, Astronomical Society of the Pacific, San Francisco, Calif, USA, 1994.

[2] M. F. Bode, Ed., Robotic Observatories, Wiley-Praxis Series in Astronomy and Astrophysics, John Wiley \& Sons, New York, NY, USA, 1995.

[3] W. P. Chen, C. Lemme, and B. Paczynski, Eds., Small Telescope Astronomy on Global Scales, vol. 246 of Astronomical Society of the Pacific Conference Series, Astronomical Society of the Pacific, San Francisco, Calif, USA, 2001.

[4] T. D. Oswalt, Ed., The Future of Small Telescopes in the New Millennium, Kluwer Academic Publishers, Dordrecht, The Netherlands, 2003.

[5] J. A. Eaton, W. Henry, and F. C. Fekel, "Advantages of automated observing with small telescopes," in The Future of Small Telescopes in the New Millennium, T. D. Oswalt, Ed., vol. 2, pp. 189-207, Kluwer Academic Publishers, Dordrecht, The Netherlands, 2003.

[6] I. G. van Breda, "Monitoring of active galactic nuclei," in Robotic Observatories, M. F. Bode, Ed., Wiley-Praxis Series in Astronomy and Astrophysics, pp. 101-105, John Wiley \& Sons, New York, NY, USA, 1995.

[7] L. Szabados, "Variable star research with small telescopes," in The Future of Small Telescopes in the New Millennium, T. D. Oswalt, Ed., vol. 3, pp. 207-223, Kluwer Academic Publishers, Dordrecht, The Netherlands, 2003.

[8] C. H. McGruder, S. B. Howell, and M. E. Everett, "The detection extrasolar planets via the transit method," in The Future of Small Telescopes in the New Millennium, T. D. Oswalt, Ed., vol. 3, pp. 173-187, Kluwer Academic Publishers, Dordrecht, The Netherlands, 2003.

[9] M. I. Andersen and H. Pedersen, "Gamma-ray burst optical follow ups with robotic telescopes," Astronomische Nachrichten, vol. 325, no. 6-8, pp. 490-495, 2004.

[10] J. Huchra, "The importance of small telescopes to cosmological research," in The Future of Small Telescopes in the New Millennium, T. D. Oswalt, Ed., vol. 3, pp. 343-354, Kluwer Academic Publishers, Dordrecht, The Netherlands, 2003.

[11] N. Gehrels, G. Chincarini, P. Giommi, et al., "The Swift gamma-ray burst mission," Astrophysical Journal, vol. 611, no. 2, pp. 1005-1020, 2004.

[12] C. Winkler, T. J.-L. Courvoisier, G. Di Cocco, et al., "The INTEGRAL mission," Astronomy \& Astrophysics, vol. 411, no. 1, pp. L1-L6, 2003.
[13] A. H. Jarrett, "Meteorology at Boyden observatory from 19801985," Monthly Notes of the Astronomical Society of South Africa, vol. 46, p. 11, 1987.

[14] P. Kubánek, M. Jelínek, S. Vítek, A. de Ugarte Postigo, M. Nekola, and J. French, "RTS2: a powerful robotic observatory manager," in Advanced Software and Control for Astronomy, $\mathrm{H}$. Lewis and A. Bridger, Eds., vol. 6274 of Proceedings of SPIE, Orlando, Fla, USA, May 2006.

[15] J. French, et al., "The Watcher robotic telescope system description," in preparation.

[16] A. Ferrero, et al., "Watcher observations of the prompt and early afterglow emission from GRB080905B," in preparation. 

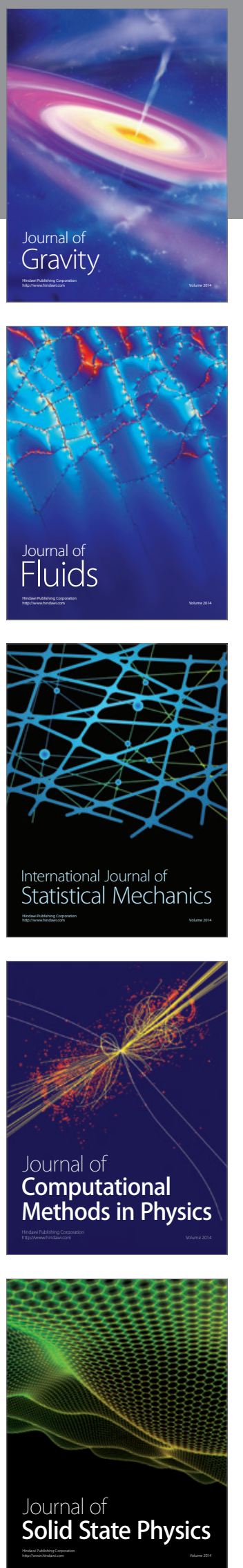

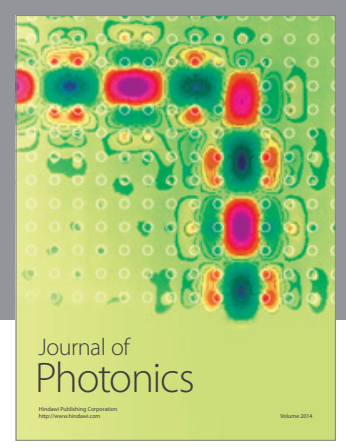

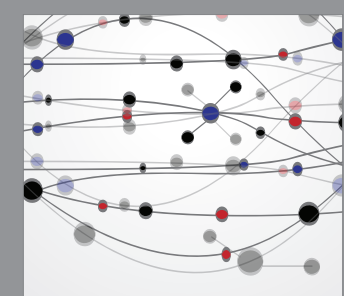

The Scientific World Journal
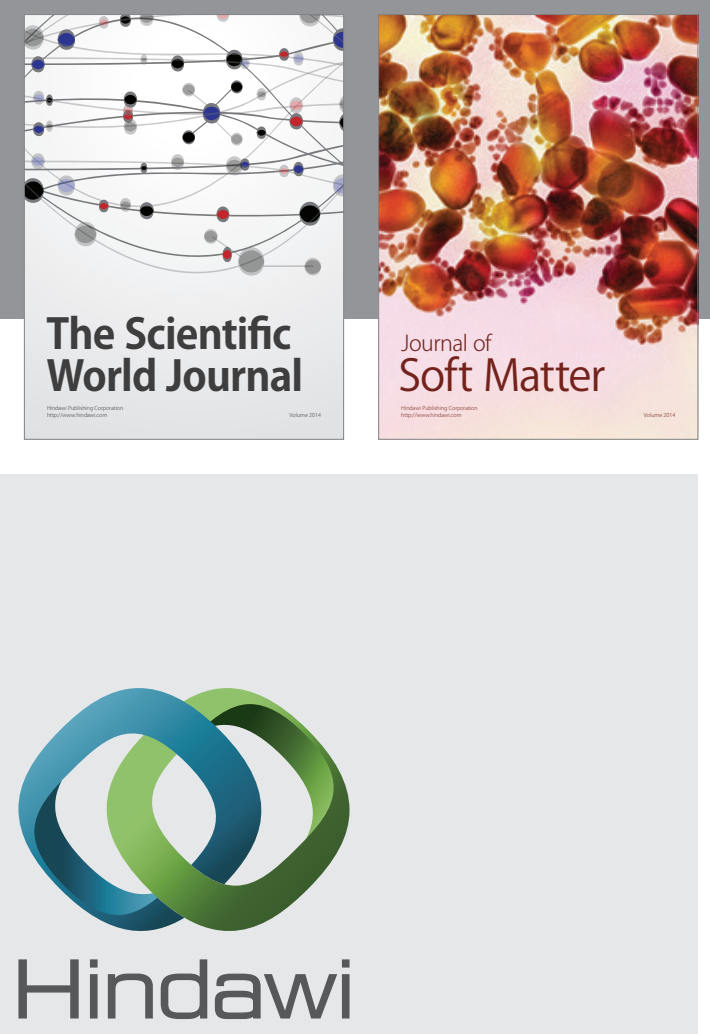

Submit your manuscripts at

http://www.hindawi.com
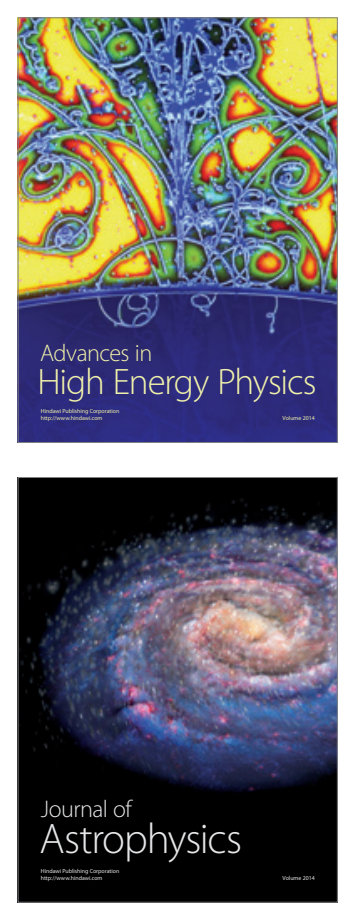
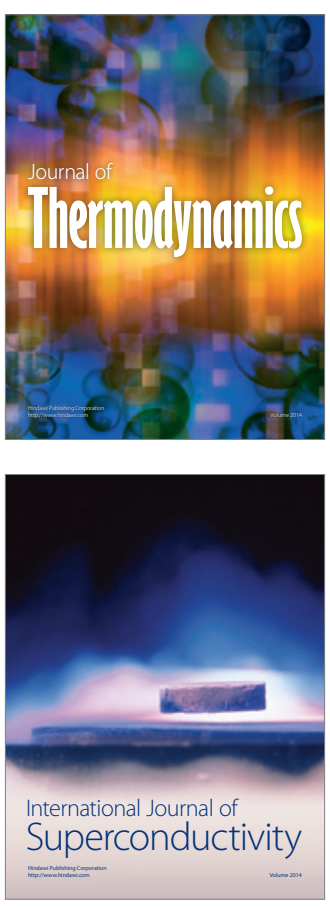
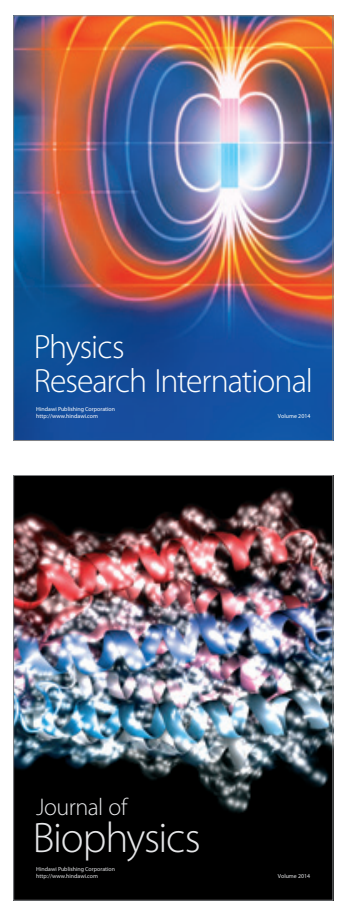
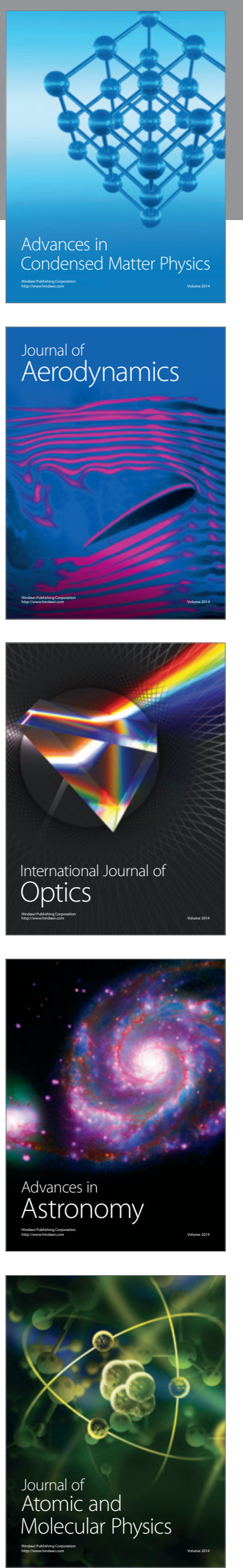